\title{
Quantitative subchondral bone perfusion imaging in knee osteoarthritis using dynamic contrast enhanced MRI
}

\author{
Bas A. de Vries ${ }^{\mathrm{a}}$, Rianne A. van der Heijden ${ }^{\mathrm{a}}$, Joost Verschueren ${ }^{\mathrm{a}, \mathrm{b}}$, Pieter K. Bos ${ }^{\mathrm{b}}$, \\ Dirk H.J. Poot ${ }^{\mathrm{a}, \mathrm{c}}$, Jasper van Tiel ${ }^{\mathrm{a}}$, Gyula Kotek ${ }^{\mathrm{a}}$, Gabriel P. Krestin ${ }^{\mathrm{a}}$, Edwin H.G. Oei ${ }^{\mathrm{a}, *}$ \\ ${ }^{a}$ Department of Radiology \& Nuclear Medicine, Erasmus MC, University Medical Center, Rotterdam, the Netherlands \\ ${ }^{\mathrm{b}}$ Department of Orthopedic Surgery, Erasmus MC, University Medical Center, Rotterdam, the Netherlands \\ ${ }^{\mathrm{c}}$ Department of Medical Informatics, Erasmus MC, University Medical Center, Rotterdam, the Netherlands
}

\section{A R T I C L E I N F O}

\section{Keywords:}

Knee osteoarthritis

Bone marrow lesions

Perfusion

dynamic contrast enhanced MRI

Quantitative MRI

\begin{abstract}
A B S T R A C T
Objective: Subchondral bone changes, characterized by increased bone turnover and vascularity, are believed to stimulate progression and pain in knee osteoarthritis (OA). The objective of this study was to evaluate the bone perfusion in knee OA using quantitative dynamic contrast enhanced MRI (DCE-MRI).

Design: Unicompartmental knee OA patients were included and underwent 3 Tesla DCE-MRI and T2weighted MRI. Quantitative DCE-MRI analysis of Ktrans and Kep, representing perfusion parameters, was performed to evaluate differences between the most and least affected knee compartment. First, DCE-MRI parameter differences between epimetaphyseal and subchondral bone in both femur and tibia were assessed. Second, DCE-MRI parameters in subchondral bone marrow lesions (BMLs) were compared to surrounding subchondral bone without BMLs.

Results: Twenty-three patients were analyzed. Median Ktrans and Kep in epimetaphyseal bone were significantly higher $(p<0.05)$ in the most affected (Ktrans: 0.014 ; Kep: $0.054 \mathrm{~min}^{-1}$ ) compared to least affected (Ktrans: 0.010; Kep: $0.016 \mathrm{~min}^{-1}$ ) compartment. For subchondral bone, DCE-MRI parameters were significantly higher $(p<0.05)$ in the most affected (Ktrans: 0.019; Kep: $0.091 \mathrm{~min}^{-1}$ ) compared to least affected (Ktrans: 0.014; Kep: $0.058 \mathrm{~min}^{-1}$ ) compartment as well. Subchondral BMLs detected on fat-saturated T2weighted images were present in all patients. Median Ktrans $\left(0.091 \mathrm{vs} 0.000 \mathrm{~min}^{-1}\right)$ and Kep $(0.258 \mathrm{vs}$ $0.000 \mathrm{~min}^{-1}$ ) were significantly higher within subchondral BMLs compared to surrounding subchondral bone without BMLs $(p<0.001)$.

Conclusions: Increased perfusion parameters in epimetaphyseal bone, subchondral bone and BMLs are observed in unicompartmental knee OA. BMLs likely account for most of the effect of the higher bone perfusion in knee $\mathrm{OA}$.
\end{abstract}

(c) 2019 Elsevier Inc. All rights reserved.

\section{Introduction}

Osteoarthritis (OA) is the most frequent form of arthritis and has major consequences for the individual patient and public health [1]. Recent insights show that $\mathrm{OA}$ is a whole organ disease in which many joint tissues are involved [2]. OA in the knee is characterized by degeneration of articular cartilage, synovial inflammation, and changes in the subchondral bone [3]. Animal studies showed that cartilage damage is one of the effects of injury to the subchondral bone, and that subchondral bone injury precedes cartilage changes $[4,5]$. Changes in subchondral bone could be a marker of altered fluid dynamics, which are thought to affect the excretion of cytokines that regulate and accelerate bone remodeling and cartilage degeneration

\footnotetext{
* Corresponding author

E-mail address: e.oei@erasmusmc.nl (E.H.G. Oei).
}

[6]. The altered fluid dynamics seems to be associated with inflammation [7]. A recent study in hip OA showed that bone marrow lesions (BMLs) on magnetic resonance imaging (MRI) are characterized by increased bone turnover and vascularity, which was confirmed by histopathology [8]. Moreover, subchondral bone changes in OA have been recognized as a key factor in the progression of OA and the perception of pain in OA patients [9-11]. Increased tissue vascularity, accompanied by increased remodeling activity, due to changes in the subchondral bone are thus characteristic for the process of $\mathrm{OA}$.

Changes in subchondral bone can be visualized using different MRI techniques. For example, T2-weighted fat-saturated MRI can be used to depict fluid containing areas in bone marrow as regions of increased signal intensity, which could indicate a BML. Subchondral bone perfusion in undifferentiated knee OA can also be visualized and quantified with gadolinium-based dynamic contrast enhanced 
MRI (DCE-MRI) [12]. Therefore, DCE-MRI holds promise to further characterize the role of subchondral bone and BMLs in the process of $\mathrm{OA}$.

DCE-MRI combined with a pharmacokinetic model enables quantitative assessment of microvascular structure and function within a tissue, expressed by DCE-MRI parameters. Various pharmacokinetic compartment models have been described, for example Tofts et al. [13] or Brix et al. [14]. All models aim to estimate physiological parameters such as blood flow, blood volume, and extravascular permeability [15]. Tofts model is widely used and it has recently been demonstrated to be the most accurate model for bone [16]. An important physiological parameter is the volume transfer constant (Ktrans), which is a measure of the volume transfer constant between blood plasma and extracellular extravascular space (EES) [17]. Another important parameter is Kep, which is the rate constant from the EES to the vascular component. Together, these two parameters provide robust quantitative outcome parameters of local tissue perfusion [18].

The goal of this study was to evaluate perfusion in bone of the osteoarthritic knee with quantitative DCE-MRI. To this end, two objectives were defined. The first objective was to compare perfusion in epimetaphyseal and subchondral bone between osteoarthritic and less osteoarthritic bone in patients with unicompartmental OA. The second objective was to evaluate perfusion in subchondral BMLs in comparison with surrounding bone tissue. Our hypothesis was that in both the osteoarthritic bone and in BMLs the DCE-MRI perfusion parameters are increased.

\section{Methods}

\section{Study population}

DCE-MRI data was acquired for a study focusing on the validation of multiple quantitative MRI techniques in OA [19]. Patients aged 18 years or older with unicompartmental (either medial or lateral) knee OA were included from the outpatient clinic of the Department of Orthopedic Surgery of Erasmus University Medical Center Rotterdam. As all patients were suffering from unicompartmental knee OA, perfusion could be compared within the same knee for osteoarthritic bone (affected) and less-affected bone. All patients were scheduled for total knee replacement because of moderate to severe (K\&L 3-4) radiographic knee OA according to Kellgren \& Lawrence [20]. Patients were excluded in case of varus or valgus deformity in the knee above $10^{\circ}$ or chondrocalcinosis. Other exclusion criteria were contraindications to undergo MRI, pregnancy, lactating women, renal insufficiency and allergy to contrast agents. The study was approved by the institutional review board of Erasmus MC (Rotterdam, The Netherlands), MEC-2012-218. Written informed consent was obtained from all subjects.

\section{Image acquisition}

Multisequence MRI was performed on a 3T MR system (Discovery MR750, General Electric Healthcare, Milwaukee, WI, USA) using a dedicated 8-channel knee transmit/receive coil. DCE-MRI was acquired in the sagittal plane, using a fat-suppressed 3D fast spoiled gradient echo (FSPGR) sequence with 35 phases of $10 \mathrm{~s}$. Intravenous contrast ( $0.2 \mathrm{mmol} / \mathrm{kg}$ Magnevist (Bayer, Germany)) was administered using a power injector with a rate of $2 \mathrm{ml} / \mathrm{s}$ started after the first phase and followed by a saline flush. The field of view (FOV) was $22 \times 22 \mathrm{~cm}$, with an in-plane resolution of $0.85 \times 1.20 \mathrm{~mm}$ and $5 \mathrm{~mm}$ slice thickness, a flip angle of $30^{\circ}$ and repetition time of $9.3 \mathrm{~ms}$ was used. The protocol also included a fat-suppressed sagittal T2weighted fast spin echo sequence with a FOV of $15 \times 15 \mathrm{~cm}, 3 \mathrm{~mm}$ slice thickness, and an in-plane resolution of $0.36 \times 0.59 \mathrm{~mm}$. No B1+ field or T1 mapping sequences were included.

\section{Image analysis}

Quantitative DCE-MRI analysis was performed using Tofts pharmacokinetic model [21]. Accordingly, the DCE-MRI perfusion parameter maps of Ktrans and Kep were determined using the DCETool in Horos [22]. The arterial input function (AIF) was determined by a region of interest in the popliteal artery. Ktrans reflects the volume transfer constant into the tissue compartment, while Kep describes the rate constant back into the vascular component [13].

For the first objective, delineation of the epimetaphyseal and subchondral bone was performed on the DCE scans where the cortical and subchondral bone could be clearly discriminated. Epimetaphyseal bone was defined as the bone reaching from the articular bone surface to the metaphyseal/diaphyseal junction. For the femur and tibia, the bone regions of interest (ROIs) were drawn on three slices of both the most affected and least affected knee compartments, selecting the central slice within both femur condyles, as well as a slice directly medial and lateral of these central slices. This resulted in a total of twelve ROIs per knee for the epimetaphyseal bone. The subchondral bone ROIs were constructed by reducing the epimetaphyseal ROIs to $1 \mathrm{~cm}$ from the articular bone surface (Fig. 1), again resulting in 12 ROIs. Both the epimetaphyseal and subchondral bone ROIs were divided into two groups, comprising the least and the most affected compartment of the knee. Subsequently, mean perfusion parameters were calculated for each compartment (femur and tibia combined) and also for the femur/tibia within compartment separately, by averaging over the ROIs in three adjacent slices. Epimetaphyseal and subchondral ROIs were delineated using the Horos software package (Horosproject.org, USA).

For the second objective, subchondral BMLs, seen as ill-defined areas of subchondral hyperintensity on fluid-sensitive sequences [23] were delineated on the fat-suppressed T2-weighted images. BMLs exhibit higher signal intensity than the surrounding bone on these T2weighted acquisitions. Cystic or partially cystic lesions were not considered BMLs in this analysis. The most clearly visible BML per patient was selected, independent of the most affected OA side. Accordingly, an elliptical shaped ROI was drawn within the maximum margins of the BML using Horos. The location of the BML could be in either the tibia or femur and one BML per patient was selected. For comparison, another ROI was drawn in subchondral bone with normal low signal intensity on the fat-suppressed T2-weighted images. Again, this resulted in two groups, comprising ROIs within BMLs and ROIs in normal bone marrow without BML and mean perfusion values over the ROIs were computed. All ROIs were drawn by a researcher with a technical medical degree and more than 3 years of experience in musculoskeletal imaging research (B.d.V.). Registration between the T2weighted images and the DCE-scan was performed to propagate the ROIs to the DCE-scan. Analysis was performed using the DCETool in Horos. An example of a T2-image, DCE perfusion map and the fused image are shown in Fig. 2. Before the perfusion parameters were calculated, the DCE-MR images were registered over time to correct for patient movement during the DCE-MRI acquisition, using an automated rigid registration tool [24].

\section{Statistical analysis}

The image analysis results in mean Ktrans and Kep values for each region in each patient. Subsequently, for each region (femur, tibia or combination of femur and tibia) within each compartment (least affected or most affected) the median Ktrans and Kep over all patients were calculated, as well as the interquartile range (IQR) as a measure of variability. The Shapiro-Wilk test was used to evaluate the normal distribution of Ktrans and Kep. A paired Wilcoxonsigned-rank test was used to compare the Ktrans and Kep values of the most affected with the least affected bone compartment for both the epimetaphyseal and subchondral bone and to compare the Ktrans 


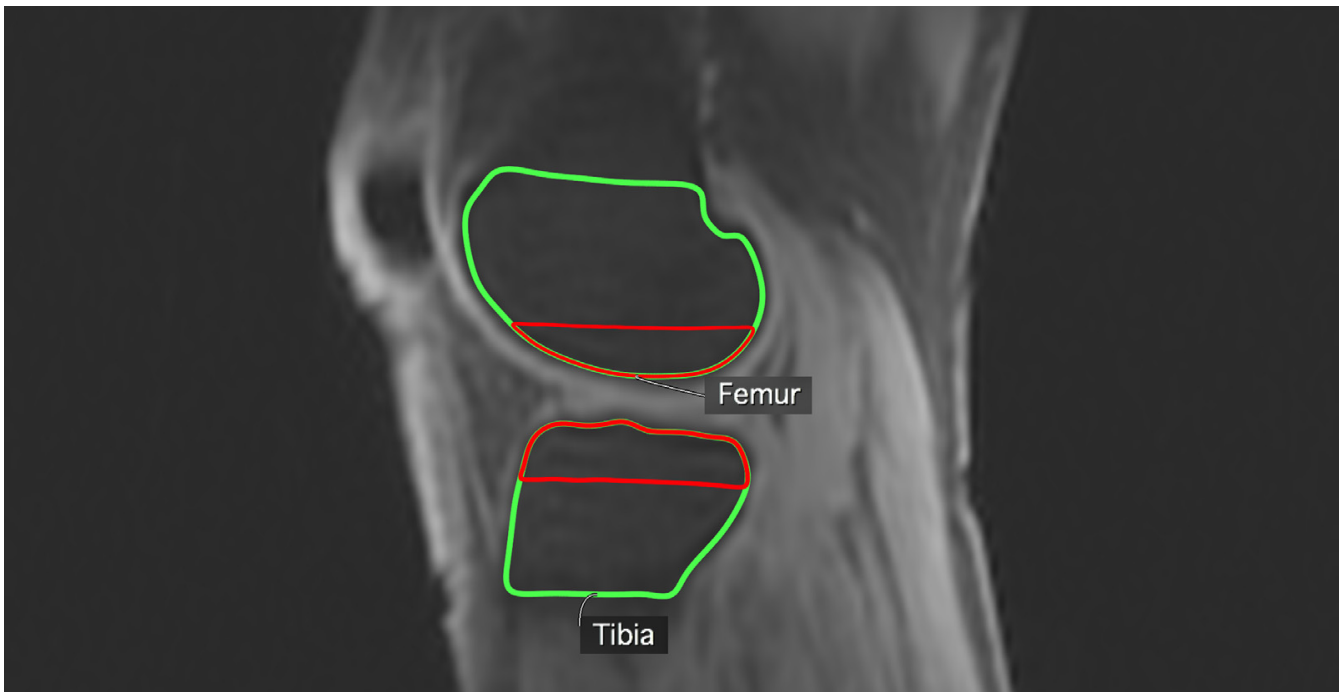

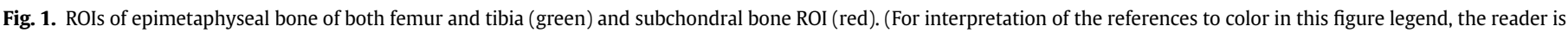
referred to the web version of this article.)

and Kep values in BML/non-BML. A non-parametric Levene's test was performed to verify the equality of variances in the samples (homogeneity of variance), i.e. to determine whether the variance between the two groups were significantly different or assumed equal $[25,26]$. A p-value of 0.05 was considered statistically significant. Statistical analysis was performed using SPSS v24 (IBM, Armonk, NY, USA).

\section{Results}

Twenty-three patients were included between December 2012 and June 2016. Data from all patients was suitable for analysis. The mean age was 63 years and the mean BMI was 29.8. The left knee was affected in 11 patients, and the right knee in 12 patients. For none of the patients a traumatic event as a direct cause of the knee OA was described in the medical records. All patient characteristics are shown in Table 1. Both the Ktrans and Kep values for all measurements showed a non-normal distribution ( $p$-value $<0.05$ ).

Table 2 shows the median and IQR values of DCE-MRI perfusion parameters of the most and least affected compartment in both epimetaphyseal and subchondral bone. These perfusion parameters were calculated in both the most affected and least affected compartment within the tibia and the femur and also for the combination of tibia and femur. Tests of the homogeneity of variances using the modified Levene's test did not reveal a violation of this assumption in the analyzed groups. Ktrans reflects the volume transfer constant into the tissue compartment, while Kep describes the rate constant back into the vascular compartment. In short, Ktrans reflects the supply of blood to bone tissue for Kep this is the opposite, i.e. perfusion from bone tissue back into the vasculature [13]. In the epimetaphyseal bone, significant differences $(p<0.05)$ were found between the most affected and least affected compartment in the Ktrans values observed in the femur, tibia, and both combined. Also for the Kep values significant differences $(p<0.05)$ between the most affected and least affected compartment were found in the tibia and both combined. For the subchondral bone, Kep and Ktrans showed statistically significant differences $(p<0.05)$ between the most affected and least affected compartment in the tibia and when combining tibia and femur.

Subchondral BMLs detected on fat-saturated T2-weighted images were present in all 23 patients. In total 23 BMLs were selected, one per patient, of which eighteen were located in the most affected compartment and five BMLs were located in the least affected

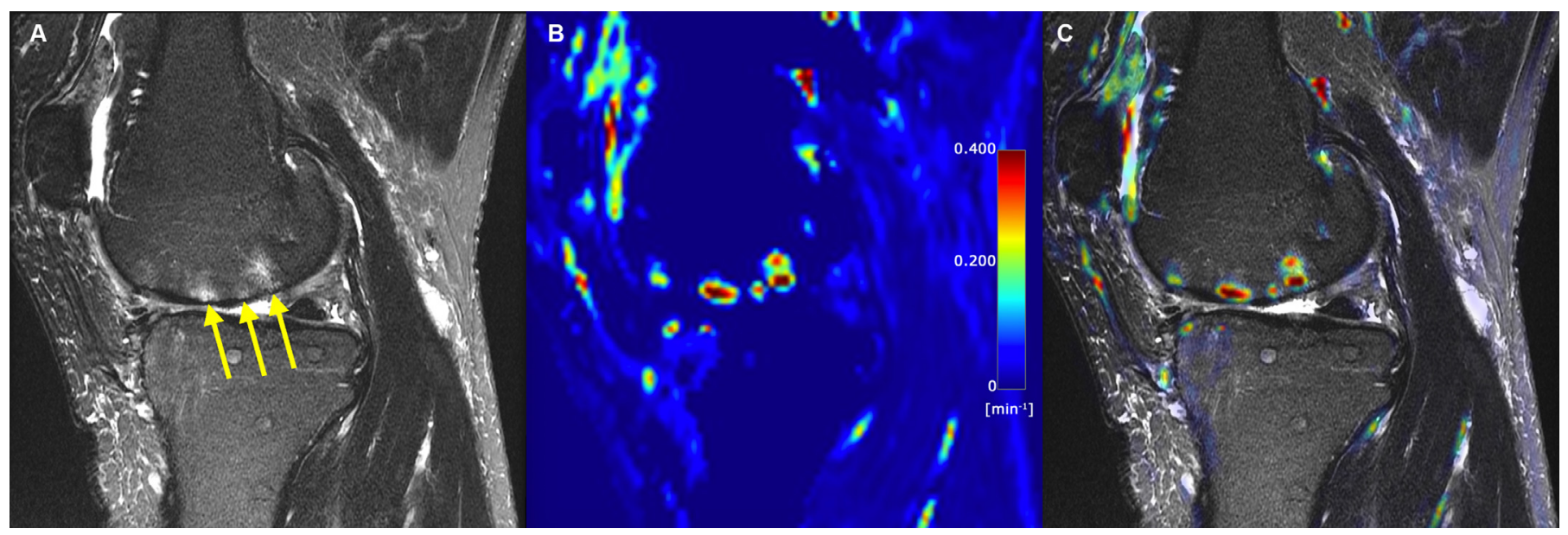

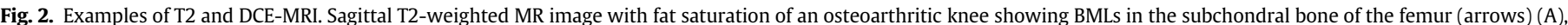

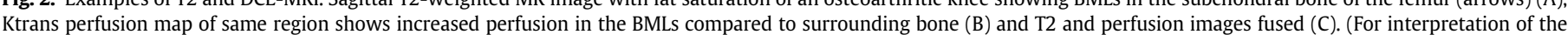
references to color in this figure legend, the reader is referred to the web version of this article.) 
Table 1

Patient characteristics

\begin{tabular}{ll}
\hline Parameter & Value \\
\hline No. of patients & 23 \\
& Males: $n=8$ \\
Mean age, y (range) & Females: $n=15$ \\
Mean BMI (range) & $63(52-73)$ \\
Knee & $29.8(21-39)$ \\
& Left $(n=11)$ \\
Most affected compartment & Right $(n=12)$ \\
& Medial $(n=19)$ \\
Most affected compartment (K\&L grade) & Lateral $(n=4)$ \\
& Grade $0(n=0)$ \\
& Grade $1(n=0)$ \\
& Grade $2(n=4)$ \\
& Grade $3(n=12)$ \\
& Grade $4(n=7)$ \\
Least affected compartment (K\&L grade) & Grade $0(n=6)$ \\
& Grade $1(n=13)$ \\
& Grade $2(n=4)$ \\
& Grade $3(n=0)$ \\
& Grade $4(n=0)$ \\
\hline
\end{tabular}

compartment. Variance between subchondral bone with and without a BML was tested equal. Median Ktrans and Kep were significantly $(p<0.001)$ higher within subchondral BMLs (Ktrans 0.091 IQR [0.058-0.158] and Kep 0.258 IQR[0.186-0.651] $\mathrm{min}^{-1}$ ) compared to surrounding subchondral bone without BMLs (Ktrans 0.000 IQR [0.000-0.001] and Kep 0.000 IQR[0.000-0.004] $\left.\mathrm{min}^{-1}\right)$. Both perfusion parameters, Ktrans and Kep, showed a median value of 0.000 in the normal subchondral bone. The IQR of both parameters was close to zero. Finally, no differences in Ktrans and Kep were observed between different locations of BMLs (tibial and femur, most affected and least affected compartment).

\section{Discussion}

In this study, perfusion parameters in bone were measured with quantitative DCE-MRI in knees with unicompartmental knee OA. The most and least affected compartment of the knee, but also areas with and without BMLs, were compared in terms of perfusion parameters. As hypothesized, this study showed that Ktrans and Kep values of both epimetaphyseal and subchondral bone were significantly higher in the most affected compared to the least affect compartment in patients with unicompartmental knee OA. In addition, subchondral BMLs were associated with higher Ktrans and Kep compared to subchondral bone regions without BMLs. Both findings were consistent with our hypothesis.

Budzik et al. recently showed that perfusion parameters were higher in OA bone compared to non-OA bone in knee OA [27]. They also showed a positive correlation with the WORMS scoring of BMLs. In their study a model free DCE-MRI analysis method was applied, which only provides a generic AUC measurement, in contrast to the current study in which quantitative parameters based on a pharmacokinetic model were used as the outcome parameters.

Another recent study from Aaron et al. [12]. studied OA bone perfusion in osteoarthritic bone in the human knee with DCE-MRI. Using in-house built software based on the Brix model, they found that the perfusion in normal and OA subchondral bone is different. Overall, they found a decrease in Kep and time-intensity-curve parameters, which is contrary to our results. Seah et al. [28] showed a correlation between the BML grade and Kel, which represents the washout of gadolinium contrast agent. Both studies did not evaluate the volume transfer constant Ktrans because they used the Brix pharmacokinetic model instead of Tofts that was used in our study. An important difference between Brix and Tofts is that in Brix there is no use of an AIF. Therefore the Ktrans parameter, a measure of the volume transfer constant between blood plasma and extracellular extravascular space, cannot be calculated in Brix, while this is considered an important physiological parameter. In a prior study it has been demonstrated that Tofts renders better results than Brix in bone [16]. In that same study it was recommended to use a groupwise or an subject specific AIF, where we chose for the latter. A fixed AIF was not possible due to the difference of arrival time of the bolus. Since a groupwise AIF method was not available within the DCE Tool, we applied a subject specific method. All AIF curves were individually visually inspected and appeared to capture the bolus peak adequately. Moreover, accurate between-subject comparisons are precluded in the Brix model, which is considered another drawback of this analysis. Another, and possibly most important, difference is that Aaron et al. selected only one, mid-coronal, ROI of only the tibial bone in each patient and that no single patient demonstrated a BML in the selected ROI. In our study 12 ROIs per patient were drawn; six in each compartment, divided over tibia and femur. The fact that they found no

Table 2

DCE-MRI perfusion parameters of the knee bone. p-values of the difference between least and most affected are reported. p-values $<0.05$ are indicated with *. IQR $=$ interquartile range

\begin{tabular}{|c|c|c|c|c|c|c|}
\hline & \multicolumn{3}{|c|}{$\mathrm{K}_{\text {trans }}\left(\min ^{-1}\right)$} & \multicolumn{3}{|c|}{$\mathrm{K}_{\mathrm{ep}}\left(\min ^{-1}\right)$} \\
\hline & Median & IQR & $p$-value & Median & IQR & $p$-value \\
\hline \multicolumn{7}{|l|}{ Epimetaphyseal knee bone } \\
\hline \multicolumn{7}{|l|}{ Femur } \\
\hline Least affected compartment & 0.010 & {$[0.002-0.024]$} & \multirow[t]{2}{*}{$0.013^{*}$} & 0.041 & {$[0.012-0.108]$} & \multirow[t]{2}{*}{0.059} \\
\hline $\begin{array}{l}\text { Most affected compartment } \\
\text { Tibia }\end{array}$ & 0.012 & [0.005-0.039] & & 0.048 & {$[0.020-0.163]$} & \\
\hline Least affected compartment & 0.009 & {$[0.003-0.017]$} & \multirow[t]{2}{*}{$0.018^{*}$} & 0.025 & {$[0.008-0.081]$} & \multirow[t]{2}{*}{$0.001^{*}$} \\
\hline Most affected compartment & 0.017 & {$[0.006-0.054]$} & & 0.061 & {$[0.013-0.172]$} & \\
\hline \multicolumn{7}{|l|}{ Femur and Tibia combined } \\
\hline Least affected compartment & 0.010 & {$[0.003-0.022]$} & \multirow[t]{2}{*}{$0.001^{*}$} & 0.016 & {$[0.007-0.047]$} & \multirow[t]{2}{*}{$<0.001^{*}$} \\
\hline Most affected compartment & 0.014 & {$[0.005-0.047]$} & & 0.054 & {$[0.016-0.165]$} & \\
\hline \multicolumn{7}{|l|}{ Subchondral knee bone } \\
\hline \multicolumn{7}{|l|}{ Femur } \\
\hline Least affected compartment & 0.007 & {$[0.002-0.023]$} & \multirow[t]{2}{*}{0.078} & 0.051 & {$[0.011-0.087]$} & \multirow[t]{2}{*}{0.346} \\
\hline $\begin{array}{l}\text { Most affected compartment } \\
\text { Tibia }\end{array}$ & 0.013 & {$[0.004-0.044]$} & & 0.064 & {$[0.019-0.200]$} & \\
\hline Least affected compartment & 0.016 & {$[0.006-0.032]$} & \multirow[t]{3}{*}{$0.045^{*}$} & 0.064 & {$[0.024-0.234]$} & \multirow[t]{3}{*}{$0.039^{*}$} \\
\hline Most affected compartment & 0.025 & {$[0.007-0.102]$} & & 0.155 & {$[0.030-0.270]$} & \\
\hline Femur and Tibia combined & & & & & & \\
\hline Least affected compartment & 0.014 & {$[0.003-0.028]$} & \multirow[t]{2}{*}{$0.007^{*}$} & 0.058 & {$[0.013-0.123]$} & \multirow[t]{2}{*}{$0.025^{*}$} \\
\hline Most affected compartment & 0.019 & {$[0.005-0.074]$} & & 0.091 & {$[0.027-0.253]$} & \\
\hline
\end{tabular}


BMLs is of concern, because it is known that in $\sim 70 \%[29,30]$ of radiographic knee OA BMLs are seen. In addition, in our study BMLs were observed in all patients.

Another strength of our study is the inclusion of a homogeneous patient population with unicompartmental knee OA. This enabled the analysis of most affected bone compartment compared to least affected bone compartment within the same joint. As analysis was performed within the same patient, the influence from possible confounders such as BMI was low. In addition, not only the perfusion of small bone regions, but also of the whole epimetaphyseal area was analyzed.

A significant difference in DCE-MRI parameters in a BML compared to subchondral bone was seen, for example the median Ktrans in a BML was $0.091 \mathrm{~min}^{-1}[0.058-0.158]$ and $0.000[0.000-0.001]$ in subchondral bone $(p<0.001)$, even in our sample of one BML selected per patient. We chose to only analyze one BML per patient, although many patients had more than one BML in their knee. The other BMLs visually showed the same increase in perfusion parameters on the whole knee perfusion maps. Since subchondral BMLs were highly associated with increased perfusion parameters compared to subchondral bone regions without BMLs, BMLs likely account for most of the effect of the increased bone perfusion in knee $\mathrm{OA}$. In fact, in bone marrow outside a BML the perfusion was almost unmeasurable in most of the subjects. An example can also be seen in Fig. 2. It is thought that this increase in perfusion may be related to inflammation [31].

As known from previous literature, also encountered in the current study, perfusion in the normal bone is low. Since the proportion of areas in which the perfusion was close to zero exceeded $50 \%$, it was not considered meaningful to use median values within the ROI even though these perfusion parameters within the ROI itself showed non-normal distribution. We therefore chose to calculate the mean values within the ROI.

For the analysis of the epimetaphyseal and subchondral regions, we did not use registration to register the T2 images to the DCE-MRI. The ROIs could be drawn directly on the DCE-MR images, because the cortical and subchondral bone could be clearly delineated. However, we did perform a rigid registration within these DCE-MR images to overcome patient movement during this dynamic scan.

A limitation of this study is the lack of longitudinal measurements. For this reason it is not possible to evaluate the effects of higher perfusion parameters in (subchondral) bone on the progression of OA. In future research, it would be very interesting to evaluate whether active BMLs with higher perfusion also show higher rates of cartilage degeneration over time in the overlying cartilage layer.

In this study we calculated Kep, which is dependent on the washout of the contrast agent [21]. Since it is possible that the end of the washout phase is not reached due to the duration of the DCE-MRI scan, we reviewed time intensity curves which demonstrated that the maximum contrast agent concentration was reached before the last phase of the DCE-MRI acquisition. Therefore, we believe Kep values to be a valid outcome parameter in our study. No B1+ and T1 correction was possible, as no $\mathrm{B} 1+$ or pre-contrast $\mathrm{T} 1$ map was acquired. A fixed T1(0) value of 1443 (standard value of the DCE Tool used in Horos) was used instead. Because of the large differences in DCE-MRI parameters observed in this study, particularly for BML versus surrounding bone marrow, we do not expect that these limitations would have changed the outcomes of this study. It is also worth noting that we used a dedicated transmit/receive knee coil with relatively homogeneous B1 field.

At the time of the MR acquisitions, linear gadolinium contrast agents, like gadopentetate dimeglumine, were commonly in use. Since then these have been withdrawn from the EU market and have been replaced by alternatives that carry less risks. As the perfusion kinetics of these alternatives is similar, we expect our results to be relevant for the newer generation contrast agents as well.
In conclusion, an increase in perfusion parameters in the epimetaphyseal bone, the subchondral bone and the BMLs is observed in unicompartmental knee OA. BMLs likely account for most of the effect of the higher bone perfusion in knee OA. This increased perfusion may be related to inflammation and might facilitate the targeted treatment for the inflammatory lesions in osteoarthritic knee bone.

\section{Declaration of Competing Interest}

None.

\section{Acknowledgments}

We thank Melek Ekinci, BSc., for assistance in preliminary data analysis.

\section{Role of the funding source}

None.

\section{Supplementary materials}

Supplementary material associated with this article can be found in the online version at doi:10.1016/j.semarthrit.2019.07.013.

\section{References}

[1] Dillon CF, Rasch EK, Gu Q, Hirsch R. Prevalence of knee osteoarthritis in the United States: arthritis data from the third national health and nutrition examination survey 1991 -94. J Rheumatol 2006;33:2271-9 doi:06/13/1011.

[2] Loeser RF, Goldring SR, Scanzello CR, Goldring MB. Osteoarthritis: a disease of the joint as an organ. Arthritis Rheum 2012;64:1697-707. doi: 10.1002/art.34453.

[3] Berenbaum F. Berenbaum-osteoarthritis as an inflammatory disease (osteoarthritis is not osteoarthrosis!). Osteoarthr Cartil 2013;21:16-21. pii: S1063-4584(12) 01025-4. doi: 10.1016/j.joca.2012.11.012.

[4] Carlson CS, Loeser RF, Jayo MJ, Weaver DS, Adams MR, Jerome CP. Osteoarthritis in cynomolgus macaques: a primate model of naturally occurring disease. J Orthop Res 1994;12:331-9. doi: 10.1002/jor.1100120305.

[5] Radin EL, Martin RB, Burr DB, Caterson B, Boyd RD, Goodwin C. Effects of mechanical loading on the tissues of the rabbit knee. J Orthop Res 1984;2:221-34. doi: 10.1002 /jor.1100020303.

[6] Lee JH, Dyke JP, Ballon D, Ciombor DM, Tung G, Aaron RK. Assessment of bone perfusion with contrast-enhanced magnetic resonance imaging. Orthop Clin North Am 2009;40:249-57. doi: 10.1016/j.ocl.2008.12.003.

[7] Scanzello CR, Loeser RF. Editorial: inflammatory activity in symptomatic knee osteoarthritis: not all inflammation is local. Arthritis Rheumatol 2015;67:2797800. doi: 10.1002 /art.39304.

[8] Shabestari M, Vik J, Reseland JE, Eriksen EF. Bone marrow lesions in hip osteoarthritis are characterized by increased bone turnover and enhanced angiogenesis. Osteoarthr Cartil 2016:24:1745-52. doi: 10.1016/j.joca.2016.05.009.

[9] Roemer FW, Guermazi A, Javaid MK, Lynch JA, Niu J, Zhang Y, et al. Change in MRIdetected subchondral bone marrow lesions is associated with cartilage loss: the MOST Study. A longitudinal multicentre study of knee osteoarthritis. Ann Rheum Dis 2009:68:1461-5. doi: 10.1136/ard.2008.096834.

[10] Felson DT, Niu J, Guermazi A, Roemer F, Aliabadi P, Clancy M, et al. Correlation of the development of knee pain with enlarging bone marrow lesions on magnetic resonance imaging. Arthritis Rheum 2007·56:2986-92 doi: 10.1002/art.22851.

[11] Felson DT, McLaughlin S, Goggins J, LaValley MP, Gale ME, Totterman S, et al. Bone marrow edema and its relation to progression of knee osteoarthritis. Ann Intern Med 2003;139. doi: 10.7326/0003-4819-139-5 Part_1-200309020-00008.

[12] Aaron RK, Racine JR, Voisinet A, Evangelista P, Dyke JP. Subchondral bone circulation in osteoarthritis of the human knee. Osteoarthr Cartil 2018. doi: 10.1016/j. joca.2018.04.003.

[13] Tofts PS, Brix G, Buckley DL, Evelhoch JL, Henderson E, Knopp M V et al. Estimating kinetic parameters from dynamic contrast-enhanced T(1)-weighted MRI of a diffusable tracer: standardized quantities and symbols. J Magn Reson Imaging 1999; 10:223-32.

[14] Brix G, Semmler W, Port R, Schad LR, Layer G, Lorenz WJ. Pharmacokinetic parameters in cns gd-dtpa enhanced mr imaging. J Comput Assist Tomogr 1991;15:6218. doi: 10.1097/00004728-199107000-00018.

[15] Sourbron SP, Buckley DL. Tracer kinetic modelling in MRI: estimating perfusion and capillary permeability. Phys Med Biol 2012;57. doi: 10.1088/0031-9155/57/2/R1.

[16] Poot DHJ, van der Heijden RA, van Middelkoop M, Oei EHG, Klein S. Dynamic contrast-enhanced MRI of the patellar bone: how to quantify perfusion. J Magn Reson Imaging 2018;47:848-58. doi: 10.1002/jmri.25817. 
[17] Chikui T, Obara M, Simonetti AW, Ohga M, Koga S, Kawano S, et al. The principal of dynamic contrast enhanced MRI, the method of pharmacokinetic analysis, and its application in the head and neck region. Int J Dent 2012;2012. doi: 10.1155/ 2012/480659

[18] Zwick S, Kopp-schneider A. Simulation-based comparison of two approaches frequently used for dynamic contrast-enhanced MRI 2010:432-42. doi:10.1007/ s00330-009-1556-6.

[19] van Tiel J, Kotek G, Reijman M, Bos PK, Bron EE, Klein S, et al. Is T1 $\rho$ mapping an alternative to delayed gadolinium-enhanced MR imaging of cartilage in the assessment of sulphated glycosaminoglycan content in human osteoarthritic knees? An in vivo validation study. Radiology 2016;279:523-31. doi: 10.1148/ radiol.2015150693.

[20] Kellgren JH, Lawrence JS. Radiological assessment of osteo-arthrosis. Ann Rheum Dis 1957;16:485-93.

[21] Tofts PS, Kermode AG. Measurement of the blood-brain barrier permeability and leakage space using dynamic MR imaging. 1. Fundamental concepts. Magn Reson Med 1991;17:357-67. doi: 10.1002/mrm.1910170208.

[22] KS. DCE Tool 2015. http://kyungs.bol.ucla.edu/software/DCE_tool/DCE_tool.html (Accessed 23 May 2018).

[23] Hunter DJ, Guermazi A, Lo GH, Grainger AJ, Conaghan PG, Boudreau RM, et al. Evolution of semi-quantitative whole joint assessment of knee OA: MOAKS (MRI osteoarthritis knee score). Osteoarthr Cartil 2011;19:990-1002. doi: 10.1016/j. joca.2011.05.004
[24] Klein S, Staring M, Murphy K, Viergever MA, Pluim JPW. Elastix : A toolbox for intensity-based medical image registration, IEEE Transactions on Medical Imaging 2010;29:196-205.

[25] Nordstokke DW, Zumbo BD. A new nonparametric levene test for equal variances Psicologica 2010.

26] Nordstokke D, Zumbo B, Cairns SL, Saklofske D. The operating characteristics of the nonparametric levene Levene test for equal variances with assessment and evaluation data, Practical Assessment, Research E' Evaluation 2011;16

[27] Budzik J-F, Ding J, Norberciak L, Pascart T, Toumi H, Verclytte S, et al. Perfusion of subchondral bone marrow in knee osteoarthritis: a dynamic contrast-enhanced magnetic resonance imaging preliminary study. Eur J Radiol 2017;88:129-34 doi: 10.1016/j.ejrad.2016.12.023.

28] Seah S, Wheaton D, Li L, Dyke JP, Talmo C, Harvey WF, et al. The relationship of tibial bone perfusion to pain in knee osteoarthritis. Osteoarthr Cartil 2012;20:1527-33. doi: 10.1016/j.joca.2012.08.025.

29] Goldsmith GM, Aitken D, Cicuttini FM, Wluka AE, Winzenberg T, Ding CH, et al. Osteoarthritis bone marrow lesions at the knee and large artery characteristics. Osteoarthritis Cartilage 2014;22:91-4. doi: 10.1016/j.joca.2013.10.022.

[30] Ip S, Sayre EC, Guermazi A, Nicolaou S, Wong H, Thorne A, et al. Frequency of bone marrow lesions and association with pain severity: results from a population-based symptomatic knee cohort. J Rheumatol 2011;38:1079-85. doi: 10.3899/jrheum.100587.

[31] Malemud CJ. The biological basis of osteoarthritis : state of the evidence. Curr Opin Rheumatol 2015;27:289-94. doi: 10.1097/BOR.0000000000000162. 